\title{
PROOF OF A FOLKLORE JULIA SET CONNECTEDNESS THEOREM AND CONNECTIONS WITH ELLIPTIC FUNCTIONS
}

\author{
JANE M. HAWKINS
}

Abstract. We prove the following theorem about Julia sets of the maps

$$
f_{n, p, \gamma}(z)=z^{n}+\frac{\gamma}{z^{p}}
$$

for integers $n, p \geq 2, \gamma \in \mathbb{C}$ by using techniques developed for the Weierstrass elliptic $\wp$ function and adapted to this setting.

Folklore connectedness theorem: If $f_{n, p, \gamma}$ has a bounded critical orbit, then $J\left(f_{n, p, \gamma}\right)$ is connected.

This is related to connectivity results by the author and others about $J(\wp)$, where $\wp$ denotes the Weierstrass elliptic $\wp$ function, especially where the period lattice has some symmetry. We illustrate several further connections between the dynamics of some specific elliptic functions and the family $f_{n, p, \gamma}$ for some values of $n$ and $p$.

\section{INTRODUCTION}

The purpose of this paper is to give a proof of a result about connectivity of Julia sets of rational maps of a certain type

$$
f_{n, p, \gamma}=z^{n}+\frac{\gamma}{z^{p}}
$$

given any integers $n, p \geq 2$ and any nonzero complex number $\gamma$.

In particular, we show that if $f_{n, p, \gamma}$ has a bounded critical orbit, then $J\left(f_{n, p, \gamma}\right)$ is connected. Some special cases of this result appear in a paper focused on iterating elliptic functions [5], and in a recent preprint [8]. To the author's knowledge, no proof of the general result has been given.

We also make connections between the iteration of elliptic functions and their truncated Laurent series, noting that connectivity results about Julia sets of Weierstrass elliptic $\wp$ functions over certain period lattices are proved in [4, 5, 10, 11, 13 . and studies of their parameter spaces appear in [13, 14. The techniques used in these papers, along with studies done on the maps of the form (11), studied copiously by Devaney and other authors (for example, [3, 6, 8) lead to a natural proof of this theorem. Since the Weierstrass elliptic $\wp$ function has a lot of classical and beautiful identities which aid in the understanding of the dynamics (used in [11] and later papers), this idea leads to many identities for the maps in the family given in (11). Of course some of these were already known and published, but we take the opportunity to give simple and general proofs, trying not to repeat any proofs

Received by the editors July 15, 2012.

2010 Mathematics Subject Classification. Primary 37F10, 37F45; Secondary 30D05, 30B99.

Key words and phrases. Connected Julia sets, complex dynamics, iterated elliptic functions.

This work was partially funded by a University of North Carolina, University Research Council Grant. 
that have already appeared in the literature on these maps. Exceptions are made for a few short proofs of results germane to the proof of the folklore connectedness theorem.

We set $\mathbb{C}^{*}=\mathbb{C} \backslash\{0\}$, and fix a lattice $\Lambda=\left\{m \lambda_{1}+n \lambda_{2}: m, n \in \mathbb{Z}, \lambda_{1}, \lambda_{2} \in\right.$ $\left.\mathbb{C}^{*}, \frac{\lambda_{1}}{\lambda_{2}} \notin \mathbb{Q}\right\} \subset \mathbb{C}$. Throughout $\mathbb{C}_{\infty}$ denotes the Riemann sphere. The Weierstrass elliptic $\wp$ function is defined by

$$
\wp_{\Lambda}(z)=\frac{1}{z^{2}}+\sum_{w \in \Lambda \backslash\{0\}}\left(\frac{1}{(z-w)^{2}}-\frac{1}{w^{2}}\right),
$$

and gives a periodic meromorphic function with periods $\{\lambda: \lambda \in \Lambda\}$. It is classical that the Laurent series expansion of $\wp_{\Lambda}$ about $z_{0}=0$ is written as

$$
\wp(u)=\frac{1}{u^{2}}+\frac{g_{2}}{20} u^{2}+\frac{g_{3}}{28} u^{4}+\cdots,
$$

where $g_{2}(\Lambda)=60 \sum_{w \in \Lambda \backslash\{0\}} w^{-4}$ and $g_{3}(\Lambda)=140 \sum_{w \in \Lambda \backslash\{0\}} w^{-6}$ [9]. Replacing every $z$ by $-z$ in equation (2), we see that $\wp_{\Lambda}$ is an even function (hence only even powers appear in the expansion); odd powers appear in the series expansion of $\wp_{\Lambda}^{\prime}$.

The derivative of the Weierstrass elliptic function is also an elliptic function which is periodic with respect to $\Lambda$, with series expansion given by

$$
\wp_{\Lambda}^{\prime}(z)=\frac{-2}{u^{3}}+\frac{g_{2}}{10} u+\frac{g_{3}}{7} u^{3}+\cdots .
$$

The coefficients $g_{2}$ and $g_{3}$ are invariants of the lattice $\Lambda$ in the sense that if $g_{2}(\Lambda)=g_{2}\left(\Lambda^{\prime}\right)$ and $g_{3}(\Lambda)=g_{3}\left(\Lambda^{\prime}\right)$, then $\Lambda=\Lambda^{\prime}$. Moreover, given any $g_{2}$ and $g_{3}$ such that $g_{2}^{3}-27 g_{3}^{2} \neq 0$, there exists a lattice $\Lambda$ having $g_{2}=g_{2}(\Lambda)$ and $g_{3}=g_{3}(\Lambda)$ as its invariants 9 .

Due to the form of the Laurent series of $\wp_{\Lambda}, \wp_{\Lambda}^{\prime}$, and hence an arbitrary elliptic function with period lattice $\Lambda$ (each is a rational function of $\wp_{\Lambda}$ and $\wp_{\Lambda}^{\prime}$ ), there are connections that can be made between the dynamics of parametrized families of elliptic functions, and these well-studied rational maps from (11),

$$
f_{n, p, \gamma}(z)=z^{n}+\frac{\gamma}{z^{p}}, \quad n, p \in \mathbb{N}, n, p, \geq 2, \gamma \in \mathbb{C}^{*} .
$$

For example, the author and Koss [11] showed that for triangular lattices (the lattice shape is the union of two equilateral triangles),

$$
\wp_{\Lambda}(u)=\frac{1}{u^{2}}+g u^{4}+O\left(u^{8}\right)
$$

near the origin, and $J\left(\wp_{\Lambda}\right)$ is connected. These maps are parametrized by $g_{3}$, and $g=g_{3} / 28$ in the series expansion.

Additionally for square lattices we have $g_{3}=0$, so we parametrize the dynamics by $g_{2}$. We have

$$
\wp_{\Lambda}(u)=\frac{1}{u^{2}}+g u^{2}+O\left(u^{6}\right)
$$

near the origin, and it can be shown that truncating the Laurent series after only two terms picks up a nontrivial amount of the dynamics; it was shown in [13, 10], and [5] that Julia sets for square lattices are connected. Moreover, Clemons showed in [5], Theorem 5.6, that maps of the form $f_{2,2, \gamma}$ with a nonrepelling periodic orbit and no Herman ring, have connected Julia sets. 
The reason the two-term approximation picks up much of the qualitative dynamics is that the symmetry in the lattices causes the coefficients in the higher powers of $u^{6}$ to be 0 much of the time, and very tiny when they are nonzero.

The techniques used to show connectivity carry over to the setting of rational maps, specifically the approximations to the Laurent series of $\wp_{\Lambda}$; we use them to prove what we will call the folklore connectedness theorem, Theorem 3.2 of this paper. The jumping-off point is the following lemma which shows that it does not matter if you consider the family of maps in equation (1) as a perturbation of $z^{n}$ as is done in [6], or as a perturbation of the map with a pole of order $p$ given by $z \mapsto z^{-p}$ which connects it to elliptic functions (see [4, Proposition 4.1.3).

We define the maps

$$
\mathcal{P}_{n, p, g}(z)=\frac{1}{z^{p}}+g z^{n} .
$$

The proof of the next result follows from writing out the left and right sides of (5).

Lemma 1.1. The map $\mathcal{P}_{n, p, g}$ is conformally conjugate to the map $f_{n, p, \gamma}$ defined in (1) with $\gamma=g^{\frac{p+1}{n-1}}$, for any choice of branch map. For each fixed $g, n, p$, the conjugating map $\phi(z)=g^{1 / n-1} z$ (choosing the same branch) satisfies

$$
\phi \circ \mathcal{P}_{n, p, g} \circ \phi^{-1}(z)=f_{n, p, \gamma}(z) .
$$

We note that, for a fixed $\gamma$, the number of distinct parameters in $g$-space for which $\mathcal{P}_{n, p, g}$ is conjugate to $f_{n, p, \gamma}$ depends on $n$ and $p$. We also note that $\mathcal{P}_{n, p, 1}=f_{p, n, 1}$.

Section 2 shows connections between the two families of maps, yielding useful identities for the family of maps given in (11). The statement and proof of the main theorem on connectedness of Julia sets is in Section 3.

\section{Preliminary Definitions And identities}

We fix $n, p$ to be integers $\geq 2$, and set $d=n+p$, the degree of the map $f_{n, p, \gamma}$ given in equation (11). Maps of this form exhibit a lot of symmetry and this has been discussed in many papers (see e.g., 6] and [3] and their references). We refer to the $d^{\text {th }}$ roots of unity as

$$
\Omega=\left\{\omega_{j}=\exp \left(\frac{2 \pi i j}{d}\right), j=0,1, \ldots, d-1\right\} .
$$

The set $\Omega^{n}=\left\{\omega_{j}^{n}\right\}_{j=0}^{d-1}$ is a subgroup of $\Omega$ containing at least two distinct elements (e.g., 1 and -1 when $n=p$ ), and at most $d$ elements (which occurs when $n$ and $p$ are relatively prime). For a nonzero complex number $\gamma$, the multiple valued function $\gamma^{1 / d}$ always has a unique value whose argument is in $\left[0, \frac{2 \pi i}{d}\right)$, and we call that the principle value of $\gamma^{1 / d}$ or the principal $d^{\text {th }}$ root of $\gamma$. In what follows, when we write $k^{1 / d}$ for any nonzero $k \in \mathbb{C}$ we mean the principal root unless otherwise specified; when $k>0$, the principal root of $k^{1 / d}$ is also positive. The following lemma follows from easy calculations and standard complex dynamics (see e.g., [1]), except for statement (4). It is a corollary of Proposition 2.1(11) which is proved below.

Lemma 2.1. The map $f_{n, p, \gamma}$ has $2 d-2$ critical points counting multiplicity as follows:

(1) $\infty$, with multiplicity $n-1$. Since $f_{n, p, \gamma}(\infty)=\infty$, it is also a critical value, $e_{\infty}$ 
(2) 0 , with multiplicity $p-1$. The point at 0 is a pole, hence maps to the critical value $e_{\infty}$.

(3) There are $d$ simple critical points $c_{j}=\omega_{j} \cdot((p / n) \gamma)^{\frac{1}{d}}, j=0, \ldots, d-1$.

(4) We label a critical point $c_{0}$ if $\arg \left(c_{0}\right) \in\left[0, \frac{\pi}{d}\right)$; we define $e_{0}=e_{0}(n, p, \gamma)=$ $f_{n, p, \gamma}\left(c_{0}\right)$ to be its critical value. The critical values are $\left\{\omega_{j}^{n}\left(e_{0}\right)\right\}$, and they may or may not be distinct for different $j$.

(5) Labelling the set of distinct critical values by $\left\{e_{k}\right\}_{k=0}^{q(n, d)-1}$, we have that $q(n, d) \geq 2$ for all values of $n, p \geq 2$ and any $\gamma \in \mathbb{C}^{*}$.

(6) There are $d$ order-one prepoles (preimages of 0 ) occurring at $p_{j}=\omega_{j}(-\gamma)^{\frac{1}{d}}$.

There are also several published statements closely related to the next result (it follows from [6], Proposition (Dynamical Symmetry)) and is proved in [16, Lemma 2. Lemma 2.2 also follows from Proposition 2.1 proved below. We define the Fatou set of a rational map $f, F(f)$ to be the open set of points $z \in \mathbb{C}_{\infty}$ on which the family of maps $\left\{f^{n}\right\}_{n \in \mathbb{N}}$ is normal near $z$. The Julia set, $J(f)$ is $\mathbb{C}_{\infty} \backslash F(f)$.

Lemma 2.2. For each fixed pair $n, p$, denote by $\Omega$ the $d=n+p$ roots of unity as above. Every map of the form $f_{n, p, \gamma}=f_{\gamma}$ satisfies:

(1) $F\left(f_{\gamma}\right)=\omega_{j} F\left(f_{\gamma}\right), j=1, \ldots, d-1$.

(2) $J\left(f_{\gamma}\right)=\omega_{j} J\left(f_{\gamma}\right), j=1, \ldots, d-1$.

(3) If $\gamma \in \mathbb{R}$, then $\overline{J\left(f_{\gamma}\right)}=J\left(f_{\gamma}\right)$ and $\overline{F\left(f_{\gamma}\right)}=F\left(f_{\gamma}\right)$, where $\bar{z}$ denotes complex conjugation and for any set $A \subset \mathbb{C}, \bar{A}=\{\bar{z}: z \in A\}$.

(4) For any $\gamma \in \mathbb{C}^{*}, \overline{J\left(f_{\gamma}\right)}=J\left(f_{\bar{\gamma}}\right)$.

The main difference between studying the dynamics of the maps $f_{n, p, \gamma}$ and parametrized families of elliptic functions is that for every lattice $\Lambda, \infty$ is not in the domain of $\wp_{\Lambda}$, but is in the Julia set. For meromorphic maps on $\mathbb{C}$ that do not extend to rational maps, the Fatou set of a function, $F(f)$ is the set of points $z \in \mathbb{C}_{\infty}$ such that $\left\{f^{n}\right\}_{n \in \mathbb{N}}$ is defined and normal in a neighborhood of $z$ and the Julia set is the complement of the Fatou set on the sphere [2]. Notice that $\mathbb{C}_{\infty} \backslash \overline{\bigcup_{n \geq 0} f^{-n}(\infty)}$ is the largest open set where all iterates are defined. If $f$ has at least one pole that is not an omitted value, then $\overline{U_{n \geq 0} f^{-n}(\infty)}$ has more than two elements. Since $f\left(\mathbb{C}_{\infty} \backslash \overline{\bigcup_{n \geq 0} f^{-n}(\infty)}\right) \subset \mathbb{C}_{\infty} \backslash \overline{\bigcup_{n \geq 0} f^{-n}(\infty)}$, Montel's theorem implies that

$$
J(f)=\overline{\bigcup_{n \geq 0} f^{-n}(\infty)} .
$$

The pole of $\wp_{\Lambda}$ at zero corresponds to the poles of $f_{2,2, g}$ (see the connection laid out in Lemma 1.1 and in Section 4), but in the first case, prepoles end up in $J\left(\wp_{\Lambda}\right)$ and, in the other, they are in $F\left(f_{\gamma}\right)$. However making the right adjustment reveals many similarities between the families, including at the level of parameter space (compare, e.g., [7, [12], and [14]).

The next result relates the value of the map at $\gamma=1$ to all other parameters $\gamma$, highlighting the nonlinear dependence on the parameter values. We denote by $S_{n, p}$ the standard map

$$
S_{n, p}(z) \equiv S(z)=z^{n}+\frac{1}{z^{p}} .
$$

The identities in the next proposition are related to some classical identities for Weierstrass $\wp$ functions. Equation (11) appears in [7]. 
For simplicity of notation, when there is no ambiguity about $n$ and $p$, we write

$$
f_{\gamma}(z)=f_{n, p, \gamma}(z)=z^{n}+\frac{\gamma}{z^{p}}
$$

Proposition 2.1. For any integers $n, p \geq 2$, any $\gamma, k \in \mathbb{C}^{*}$, and setting $d=n+p$, the following identities hold for $f_{\gamma}$ and for all $z \in \mathbb{C}$,

$$
\begin{aligned}
f_{\gamma^{d}}(\gamma z) & =\gamma^{n} S(z), \\
f_{\gamma}\left(\gamma^{1 / d} z\right) & =\gamma^{n / d} S(z), \\
f_{k \gamma}\left(k^{1 / d} z\right) & =k^{n / d} f_{\gamma}(z), \\
f_{\gamma}\left(\omega_{j} z\right) & =\omega_{j}^{n} f_{\gamma}(z) .
\end{aligned}
$$

There are analogous identities for the derivative of $f_{\gamma}$, following from this basic one

$$
f_{\gamma^{d}}^{\prime}(\gamma z)=\gamma^{n-1} S^{\prime}(z)
$$

Proof.

$$
\begin{gathered}
f_{\gamma^{d}}(\gamma z)=(\gamma z)^{n}+\gamma^{d} /(\gamma z)^{p} \\
=\gamma^{n} z^{n}+\gamma^{n} \gamma^{p} / \gamma^{p} z^{p}=\gamma^{n}\left(z^{n}+1 / z^{p}\right) .
\end{gathered}
$$

Then (9) - (11) follow immediately from (8).

Letting $m_{\gamma}(z)=\gamma \cdot z$,

$$
\begin{aligned}
\frac{d}{d z}\left[f_{\gamma^{d}} \circ m_{\gamma}\right](z) & =f_{\gamma^{d}}^{\prime}(\gamma z) \cdot \gamma \\
& =\gamma\left((\gamma z)^{n-1}-\frac{p \gamma^{d}}{(\gamma z)^{p+1}}\right) \\
& =\gamma^{n} z^{n-1}-\frac{p \gamma^{d+1}}{\gamma^{p+1} z^{p+1}} \\
& =\gamma^{n}\left(z^{n-1}-\frac{p}{z^{p+1}}\right) \\
& =\gamma^{n} S^{\prime}(z) .
\end{aligned}
$$

Therefore (12) holds.

Using Proposition 2.1 we obtain several results about special critical points and values.

Proposition 2.2. For integers $n, p \geq 2$, consider the maps $f_{\gamma}$ as defined in (7) and (11). Then the following hold:

(1) Choosing $\gamma=\frac{n}{p}$, for the map $f_{\gamma}$, we have $c_{0}(\gamma)=1, c_{j}(\gamma)=\omega_{j}$ and $e_{0}(\gamma)=1+\frac{n}{p}$. (The critical points are the set $\Omega$.)

(2) There exists a unique number $\kappa=\gamma(n, p)>0$ such that the real critical value of $f_{\kappa}, e_{0}(\kappa)=1$. In particular,

$$
\kappa=\left(\frac{n}{d}\right)\left(\frac{p}{d}\right)^{p / n}
$$

(The critical values are $m^{\text {th }}$ roots of unity for some $m \geq 2$.)

(3) For each $j=0, \ldots, d-1$, let $\rho_{j}=\rho_{j}(\gamma)=\left\{t c_{j}(\gamma): t>0\right\}$ denote the ray in the direction of the critical point $c_{j}$. Letting $\mathfrak{R}_{\gamma}=\left\{\rho_{j}\right\}_{j=0}^{d-1}$ denote the set of critical rays, we have

$$
f\left(\Re_{\gamma}\right) \subset \Omega^{n} \cdot \mathfrak{R}_{\gamma}=\left\{\omega \cdot z: \omega \in \Omega^{n}, z \in \rho_{j}(\gamma)\right\} \subset \mathfrak{R}_{\gamma^{n}} .
$$


Proof. Fix $(n, p)$. The first statement follows from Lemma 2.1(3). We consider the map $S(z)$ from equation (6). Its positive critical point is the positive real root of $z^{d}=\frac{p}{n}$; we denote it by $b_{0}=(p / n)^{1 / d}$. Then the critical value

$$
e_{0}=e_{0}(1)=S\left(b_{0}\right)=\left(\frac{p}{n}\right)^{\frac{n}{d}}+\left(\frac{n}{p}\right)^{\frac{p}{d}} \geq 1 .
$$

Using Proposition 2.1(9), we set $\kappa=e_{0}^{-\frac{d}{n}}$, choosing the real $n^{\text {th }}$ root.

Then

$$
f_{\kappa}\left(c_{0}(\kappa)\right)=f_{\kappa}\left(\kappa^{1 / d} b_{0}\right)=\kappa^{\frac{n}{d}} S\left(b_{0}\right)=\kappa^{\frac{n}{d}} e_{0}=1 .
$$

One can verify directly that $\kappa^{n / d} e_{0}=1$ if we use the formula given for $\kappa$ in (2): setting $\kappa=\left(\frac{n}{d}\right)\left(\frac{p}{d}\right)^{p / n}$, we have that

$$
\kappa^{\frac{n}{d}}=\left(\frac{n}{d}\right)^{\frac{n}{d}} \cdot\left(\frac{p}{d}\right)^{\frac{p}{d}}
$$

and (2) follows easily by multiplying equations (13) and (14). To prove (3), we note that by Proposition 2.1 .

$$
f_{\gamma}\left(c_{j}\right)=\omega_{j}^{n} f_{\gamma}\left(c_{0}\right)=\omega_{j}^{n} e_{0}(\gamma)=\omega_{j}^{n} \alpha^{n / d} e_{0}(\kappa)=\omega_{j}^{n} \alpha^{n / d}=\omega_{j}^{n}\left(\alpha^{n}\right)^{1 / d},
$$

where $\alpha=\gamma / \kappa$. Since $\alpha$ is a positive multiple of $\gamma$, for some $t>0, f_{\gamma}\left(c_{j}\right)=\omega_{j}^{n} t c_{i}$ for some $i$.

We now give a general formula for the critical values.

Corollary 2.3. The critical values of $f_{\gamma}$ are $e_{k}=\left(\frac{d}{p}\right) c_{j}^{n}$, where $c_{j}=c_{j}(\gamma), j=$ $0, \ldots, d-1$ are the critical points.

Proof. We use Proposition 2.1 and Proposition 2.2(1) to see that: $c_{j}(\gamma)=\gamma^{1 / d} c_{j}(n / p)$ $=\gamma^{1 / d} \omega_{j}$; then we calculate the critical values,

$$
f_{\gamma}\left(c_{j}(\gamma)\right)=\gamma^{n / d} f_{n / p}\left(c_{j}(n / p)\right)=\gamma^{n / d} \omega_{j}^{n}\left(\frac{d}{p}\right)=c_{j}(\gamma)^{n} \frac{d}{p} .
$$

We can control the postcritical behavior of $f_{n, p, \gamma}$ and hence the dynamics using the identities proved above. We note for example that whenever $n=p$, the value $\gamma=\frac{1}{4}$ always yields a critical value 1 .

Corollary 2.4. For any fixed $n, p$, and any $T>0$ the critical values of $f_{n, p, \gamma}$ satisfy $\left|e_{j}\right|=T$ if $\gamma=\kappa g$, with $g=T^{\frac{d}{n}}$ and $\kappa$ the value from Proposition 2.2 .

We obtain a precise count on the number of critical values.

Corollary 2.5. For $f_{n, p, \gamma}$, let $r=G C D(n, p)$, then $q(n, p)=d / r=\left|\Omega^{n}\right|$ (where the set of distinct critical values is denoted $\left\{e_{k}\right\}_{k=0}^{q(n, p)-1}$ as in Lemma 2.1). When $\gamma>0$, the angles of the critical values, i.e., $\left\{\frac{e_{k}}{\left|e_{k}\right|}\right\}_{k \geq 0}$, form a nontrivial multiplicative subgroup of the $d^{\text {th }}$ roots of unity.

Proof. We use Corollary 2.3 and the basic arithmetic properties of roots of unity. 
Example 2.6. The results above allow us to find explicit parameter values for a variety of dynamical behavior. We give a few examples:

(1) For fixed $n, p \geq 2$, with $d=n+p$, we can find $\gamma=k$ that has a critical point as a critical value by solving

$$
\left(\frac{p}{n}\right)^{1 / d} k^{1 / d}=\left(\frac{d}{p}\right)^{n / d} k^{n / d}
$$

for $k$. We then see that whenever

$$
k=\left(\frac{d}{p}\right)^{d /(1-n)} \frac{n}{p},
$$

we obtain a positive critical point for $f_{\gamma}, c_{0}=\left(\frac{p}{d}\right)^{1 /(n-1)}$, which is also a fixed point. Hence by taking all the $n-1$ roots, (15) and (16) are used to obtain the centers of the cardioids of the principal Mandelbrot sets as studied in many papers, including [3], where a different equivalent formula for (16) is given without proof.

(2) An example to illustrate (1) occurs if we set $n=2, p=5$, and choose

$$
\gamma=\frac{2 \cdot 5^{6}}{7^{7}}
$$

we see that the real critical point $c_{0}=\frac{5}{7}$ is fixed, and all the rest of the critical points of the form $c_{j}=\omega_{j} \cdot \frac{5}{7}, j=1, \ldots, 6$ are periodic of period 3 .

(3) Using again the values $n=2, p=5$ and applying Lemma 2.1(6), choosing

$$
\gamma=-\left(\frac{2^{2} 5^{5}}{7^{7}}\right)
$$

we obtain critical values that land on the pole at 0 under $f_{\gamma}$.

(4) With the same values of $n$ and $p$ we can solve explicitly for a value of $\gamma \approx$ -.17068 , using Corollary 2.4 (and a messier formula), such that $f_{\gamma}\left(e_{0}\right)=e_{0}$ and $f_{\gamma}^{\prime}\left(e_{0}\right)>2.5$.

There is a lot of symmetry within each Fatou component as well and this has been discussed in many papers (e.g., [7]). We prove a lemma which follows from a result proved in ([7, Symmetry Lemma).

Lemma 2.7. Suppose $U$ is a Fatou component of $f_{\gamma}$ containing two distinct critical points $c_{i}$ and $c_{k}$, but does not contain either 0 or $\infty$. Then all critical points $c_{j}, j=0, \ldots, d-1$ are in $U, U$ contains a closed loop surrounding the origin, and $U$ is not simply connected. Additionally, all the critical values $\left\{e_{0}, e_{1}, \ldots, e_{q(n, p)-1}\right\}$ are contained in one Fatou component.

Proof. Suppose $c_{j}=\omega_{m} c_{i}$ and $c_{i}$ lie in $U, m \geq 1$. We let $\alpha:[0,1] \rightarrow \mathbb{C}$ be a continuous curve in $U$ such that $\alpha(0)=c_{i}$ and $\alpha(1)=c_{j}$. We now consider $\omega_{m} \alpha$ defined by $\omega_{m} \alpha(t)=\omega_{m} \cdot \alpha(t)$. This curve goes from $c_{j}$ to $c_{j+m}$, lies in the Fatou set by symmetry, so lies in $U$ since $c_{j} \in U$. We concatenate the curves $\alpha, \omega_{m} \alpha, \omega_{m}^{2} \alpha, \cdots, \omega_{m}^{d-1} \alpha$ to obtain a closed curve $\tilde{\alpha}$ starting and ending at $c_{i}$, and staying a bounded distance away from 0 and $\infty$ (since they are each in the center of an open ball not intersecting $U$ ). Note that $\alpha$ might wind around the origin a few times, depending on $n, p, i, j$, and $k$. 
Clearly $f_{\gamma}(\tilde{\alpha})$ is a closed curve which contains all the critical values, and lies in a single component of $F\left(f_{\gamma}\right)$, namely $f_{\gamma}(U)$.

While in its original appearance in [7, Lemma 2.7 was used to prove the existence of McMullen domains, we use it to rule out certain types of behavior in the proof of Theorem 3.2. We note that the image curve $f_{\gamma}(\tilde{\alpha})$ in the proof of Lemma 2.7 could lie in the Fatou component containing 0, and this can result in a "Cantor set of circles" in the Julia set described in [7].

\section{Connectedness of the Julia set}

In this section we prove the folklore connectedness theorem for Julia sets of the form given in (11). The theorem has been stated and used quite often, but only proved in some special cases. Again, throughout this section we fix arbitrary integers $n, p \geq 2$. We begin with a few observations about the valency of points for $f_{n, p, \gamma}$. From the discussion above, there are exactly $n+p+2$ distinct critical points of $f_{\gamma} \equiv f_{n, p, \gamma}$ (defined in equations (11) and (77)). Let $v\left(z_{0}\right)$ denote the valency of $f_{\gamma}$ at $z_{0}$; i.e., the number of solutions of $y=f_{\gamma}\left(z_{0}\right)$ at $z_{0}$. Let $c_{0}, \ldots, c_{d-1}$ be the critical points defined in Lemma 2.1(3), so $v\left(c_{j}\right)=2$ for each $j$. Then we also have that $v(0)=p$ and $v(\infty)=n$. For all other $z \in \mathbb{C}, v(z)=1$.

We use the following version of the Riemann-Hurwitz relation.

Proposition 3.1 (see [1]). Let $U \neq \mathbb{C}$ be a simply connected domain bounded by a Jordan curve, and let $W$ be a connected component of $f_{\gamma}^{-1}(U)$. Suppose there are no critical values of $f_{\gamma}$ on $\partial U$. Then there exists an $m \in \mathbb{N}$ such that $f_{\gamma}$ is an $m$-fold map of $W$ onto $U$ and

$$
\chi(W)=m-\delta(W),
$$

where $\delta(W)=\sum_{z \in W}(v(z)-1)=\sum_{\text {crit pts } c \in W}(v(c)-1)$ and $\chi(W)$ is the Euler characteristic of $W$.

Corollary 3.1. For a rational map of the form $f_{\gamma}$ of degree $d$, assume that no two critical points of the form $c_{j}, j=0, \ldots, d-1$ lie in the same component of $F\left(f_{\gamma}\right)$. Let $U$ be a simply connected domain contained in $F\left(f_{\gamma}\right)$, bounded by a Jordan curve and suppose there are no critical values of $f_{\gamma}$ on $\partial U$. Let $W$ be a connected component of $f_{\gamma}^{-1}(U)$.

(1) If there are no critical values in $U$, then $f_{\gamma}^{-1}(U)$ consists of $d$ disjoint homeomorphic copies of $U$.

(2) If there is one critical value $u_{1}$ in $U$, coming from one (or more) distinct simple critical points, then $f_{\gamma}^{-1}(U)$ consists of s simply connected regions, $W_{0}, \ldots, W_{s-1}$, each a homeomorphic image of $U$ bounded by a Jordan curve, with $W_{k}$ containing no critical point and $V_{0}, \ldots, V_{t-1}$, simply connected domains, such that $f_{\gamma}$ maps each $V_{t}$ onto $U$ by a 2-fold ramified covering map (and each $V_{k}$ contains a simple critical point of the form $c_{j}$ ).

(3) If the critical value $\infty \in U$, then $U$ contains the image of the multiple critical points 0 and $\infty$ (of multiplicity $p-1$ and $n-1$ respectively). Then $W=f_{\gamma}^{-1}(U)$ consists of two simply connected regions, $W_{0}$ containing 0 and $W_{1}$ containing $\infty$; then $f_{\gamma}$ gives a p-fold branched cover of $U$ by $W_{0}$ and an $n$-fold branched cover of $U$ by $W_{1}$. 
Proof. (1) is straightforward since $f_{\gamma}$ is $d$-to-one. To show (2), if $U$ contains one critical value, then by hypothesis each component $W \subset f_{\gamma}^{-1}(U)$ must have zero or one critical point in it. If $W$ contains no critical points, then $f$ is a univalent map from $W$ to $U$, and $W$ is simply connected. If $W$ contains one critical point, then it is simple so $f_{\gamma}$ gives a 2-fold ramified cover of $U$ by $W$ and by Proposition 3.1, $\chi(W)=1$, proving (2). To see that (3) holds, under the hypotheses given, there are 2 possibilities: either there is one component of $f_{\gamma}^{-1}(U)$, call it $W$, and then $W$ necessarily contains both 0 and $\infty$, or there are two components, $W_{0}$ containing 0 , and $W_{1}$ containing $\infty$. Assuming $W=f_{\gamma}^{-1}(U)$ is connected, by the RiemannHurwitz formula from Proposition 3.1 .

$$
\chi(W)=(n+p)-((n-1)+(p-1))=2 .
$$

However, this implies that $W$ is the sphere, which is impossible since $J\left(f_{\gamma}\right) \neq \emptyset$.

Therefore the second possibility gives (3) since

$$
\chi\left(W_{0}\right)=p-(p-1)=1, \quad \text { and } \quad \chi\left(W_{1}\right)=n-(n-1)=1 .
$$

We now turn to the proof of the folklore connectedness theorem, which has been alluded to many times in the literature, but for which no proof is found. This result, together with the trichotomy theorem for escaping critical points [7], gives a complete picture of the connectivity of $J\left(f_{n, p, \gamma}\right)$ for all integers $n, d \geq 2$. A proof of the connectivity when $n=p=2$ appeared in [4] and [5], and using a different method, can be found in a preprint [8] that proves it for any $n=p \geq 2$.

Theorem 3.2 (folklore Julia set connectedness theorem). Assume $n, p \geq 2$ are integers, and

$$
f_{\gamma}(z) \equiv f_{n, p, \gamma}(z)=z^{n}+\frac{\gamma}{z^{p}}
$$

If there is a bounded critical orbit, then $J\left(f_{\gamma}\right)$ is connected.

Proof. Let $\mathcal{A}_{\infty}$ denote the attracting basin of the superattracting fixed point at $\infty$, and let $\mathcal{B}_{\infty}$ be the immediate attracting basin (the component of $\mathcal{A}_{\infty}$ containing $\infty$.) Set $d=n+p$ and let $c_{j}, j=0, \ldots, d-1$ denote the nonzero critical points in $\mathbb{C}$. Recall that if there is one bounded critical orbit, then all of the $c_{j}$ 's have bounded orbits by Lemma 2.1(3) and Proposition 2.1(11).

Step 1 . We show first that if $\left\{c_{j}\right\}_{j=0}^{d-1} \cap \mathcal{A}_{\infty}=\emptyset$, then every component of $\mathcal{A}_{\infty}$ is simply connected. Consider any closed loop $l \subset \mathcal{A}_{\infty}$ such that $l$ is contained in a component $A \subset \mathcal{A}_{\infty}$. By definition of $\mathcal{A}_{\infty}$, there exists a small open ball $U \subset \mathcal{B}_{\infty}$ and a $k \in \mathbb{N}$ such that $f_{\gamma}^{k}(l) \subset U$; by making $U$ smaller if needed (and $k$ larger), we assume that $\partial U$ contains no critical values and on $U, f$ is locally conjugate to $z \mapsto z^{n}$.

We now apply Corollary 3.1 (3) to each component of $f_{\gamma}^{-1} U$; let $W_{1}$ be the component containing $\infty$ and $W_{0}$ the component of $f_{\gamma}^{-1} U$ containing 0 . By the RiemannHurwitz formula, both are simply connected. We now proceed by induction on $j$ to show that all components of $f_{\gamma}^{-j}(U)$ are simply connected for $j=1, \ldots k$. Given a simply connected component $W$ of $f_{\gamma}^{-j}(U)$, only (1) or (3) of Corollary 3.1 can occur, and one of them must occur. In either case, $W$ is simply connected. This implies that $l$ shrinks to a point in $A$.

Step 2. Suppose next that there are no nonrepelling cycles for $f_{\gamma}$ other than the fixed point at $\infty$. This assumption, together with the hypothesis that there is a 
bounded critical orbit implies that $F\left(f_{\gamma}\right)=\mathcal{A}_{\infty}$ and that a critical point $c_{j} \in J\left(f_{\gamma}\right)$ for some $c_{j}$ (otherwise it would be attracted to $\infty$ ). Then every $c_{i} \in J\left(f_{\gamma}\right), i=$ $0, \ldots, d-1$. By Step 1 , all components of $F\left(f_{\gamma}\right)$ are simply connected so $J\left(f_{\gamma}\right)$ is connected.

Step 3. The only other possibility is that there is a nonrepelling cycle in addition to the fixed point at $\infty$. Then the cycle lies in $\mathbb{C}$, and we have one of the following: (1) an attracting (or superattracting) periodic cycle; (2) a parabolic periodic cycle; or (3) a cycle of Siegel disks. The existence of a Herman ring was ruled out in [16, with a different proof for the case $n=p$ in [8].

By symmetry of the Julia and Fatou sets in Lemma 2.2 at most one of these types can occur (although there can be more than one cycle and the periods of different cycles might not all be the same). Therefore each of the critical points $c_{j}$ is associated with a nonrepelling cycle, and there is only one type of (1)-(3) for all the $c_{j}$. By working with a higher iterate of $f_{\gamma}$ if necessary, and since $J\left(f_{\gamma}^{m}\right)=J\left(f_{\gamma}\right)$ for all $m \geq 1$, we assume $f_{\gamma}$ has a nonrepelling fixed point (or several of them) of types (1), (2), or (3).

Consider any component $B \subset F\left(f_{\gamma}\right) \backslash \mathcal{A}_{\infty}$ and a closed loop $l \subset B$. Since $B$ must be preperiodic, there is an integer $k$ such that $f_{\gamma}^{k}(l) \subset U$, where $U$ is simply connected, forward invariant, and is either an immediate basin of attraction for an attracting cycle, a Leau domain for a periodic parabolic point or a Siegel disk in a cycle of Siegel disks. We can assume by making $U$ smaller that $\partial U$ contains no critical value.

Step 4. If $f_{\gamma}$ has a Siegel disk $S$, then $S$ is simply connected and we can assume that $U=S$. Therefore $\left.f_{\gamma}\right|_{U}$ is conjugate to an irrational rotation. Then $U$ cannot contain any critical point (because irrational rotation is univalent) or critical value because the boundary of the Siegel disk is contained in the closure of the forward orbits of some critical point and therefore there must be at least one critical value $e_{i}$ in the Julia set. By symmetry none of the $e_{j}$ 's can approach $\partial U$ from inside $F\left(f_{\gamma}\right)$ [15]. Therefore $U$ contains no critical values. Moreover all $c_{j}$ must lie in $J\left(f_{\gamma}\right)$ and therefore by Corollary 3.1 (1) and Step 1, all components of $F\left(f_{\gamma}\right)$ are simply connected and the theorem holds.

Step 5. Claim 1. Each connected component in $F\left(f_{\gamma}\right) \backslash \mathcal{A}_{\infty}$ contains at most one $c_{j}$ and at most one $e_{k}$. We defer the proof of Claim 1 to Step 6. Assuming that Claim 1 holds, we show using induction on $k$ that each component $f_{\gamma}^{-k}(U)$ is simply connected. The case $k=0$ holds since $U$ is simply connected. We now assume that all components of $f_{\gamma}^{-k}(U)$ are simply connected; consider one component $U_{0} \subset f^{-k}(U)$, which by assumption is simply connected. If $U_{0}$ contains no critical value, then by Corollary $3.1(1), f_{\gamma}^{-1}\left(U_{0}\right)$ consists of $d$ homeomorphic images of $U_{0}$ so each is simply connected. If $U_{0}$ contains $e_{j}$, for some $j$, then by the claim this is the only critical value and it is the image of some simple critical points $c_{i}$. Then by Corollary 3.1(2) we have that each component of $f_{\gamma}^{-1}\left(U_{0}\right)$ is simply connected. By adjusting the original choice of $U$ a little (and at most finitely many times), we can assume that each simply connected component of $f_{\gamma}^{-j}(U)$ contains no critical value in its boundary and contains either 0 or 1 critical value in it, so we repeat the argument to see that every component of $f_{\gamma}^{-k}(U)$ is simply connected, so $l$ shrinks to a point and $J\left(f_{\gamma}\right)$ is connected, and the theorem is proved. 
Step 6. Proof of Claim 1. If $F\left(f_{\gamma}\right)=\mathcal{A}_{\infty}$, the claim is trivially true. Herman rings do not exist for $f_{\gamma}$, and the claim was proved under the assumption that there is a cycle of Siegel disks for $f_{\gamma}$ in Step 4. Therefore by Step 3, it suffices to assume there is an attracting or parabolic cycle with forward periodic Fatou component $C$; by passing to a higher iterate, assume that $f_{\gamma}(C)=C$. We know that $C$ contains at least one critical point (see for example [15, Theorems 8.5 and 10.15); suppose it has more than one $c_{j}$. Then $C$ contains all of the critical points by Lemma 2.7 and is not simply connected. Moreover there is a closed continuous loop $\alpha \subset C$ going around the origin and passing through every $c_{j}$; it can be chosen to be invariant under multiplication by any $\omega_{j}$ by Lemma 2.7. Because 0 and $\infty$ are in $\mathcal{A}_{\infty}$, and $\mathcal{A}_{\infty}$ is open, there exists some $\epsilon>0$ such that diameter $(\alpha)>\epsilon$. Let $p$ be the attracting (or parabolic) fixed point associated to $C ; p \in \bar{C}$. Since $\alpha$ is compact and $C$ is forward invariant,

$$
\lim _{n \rightarrow \infty} \sup _{z \in \alpha}\left|f_{\gamma}^{n}(z)-p\right|=0 .
$$

Recall that $\mathfrak{R}_{\gamma}=\left\{\rho_{j}\right\}_{j=0}^{d-1}$ are the critical rays from Proposition 2.2(3). Since $p \notin \mathcal{A}_{\infty}$, set

$$
\eta=\max _{\rho_{j} \in \Re_{\gamma}}\left|p-\rho_{j}\right|>0
$$

where

$$
\left|p-\rho_{j}\right|=\inf _{z \in \rho_{j}}|p-z| \geq 0 .
$$

Note that $\left|p-\rho_{j}\right|=0$ if and only $p \in \rho_{j}$. Then if $i \neq j,\left|p-\rho_{i}\right| \geq \eta(d, \epsilon)>0$, so that $\eta>0$ in equation (19). We show by induction on $k$ that for every $k, f_{\gamma}^{k}(\alpha)$ contains a point on every critical ray; i.e., $f_{\gamma}^{k}(\alpha) \cap \rho_{j} \neq \emptyset$ for each $j$. This statement is true for $k=0$; assume that $\alpha_{k} \equiv f_{\gamma}^{k-1}(\alpha)$ contains a point on every critical ray. Then by Proposition 2.2 $f_{\gamma}$ maps the critical rays into $\Omega^{n} \mathfrak{R}_{\gamma} \subset \mathfrak{R}_{\gamma^{n}}$, so $f_{\gamma}\left(\alpha_{k}\right)$ must pass through every critical value ray, which then means every critical ray. This holds because the critical rays lie on or between the critical value rays, there are at least two critical value rays, and their angles are evenly spaced around the circle (they could be negatives of each other). Using continuity of $f_{\gamma}$ on $\alpha_{k}$ and symmetry of $F\left(f_{\gamma}\right)$, the path $f_{\gamma}\left(\alpha_{k}\right)=f_{\gamma}^{k}(\alpha)$ intersecting two distinct critical value rays and not intersecting $\mathcal{A}_{\infty}$, takes on every angular value between 0 and $2 \pi$. Therefore using equation (19), $\max _{z \in \alpha}\left|f_{\gamma}^{k}(\alpha)-p\right| \geq \eta>0$, contradicting equation (18). Hence there is at most one critical point in $C$ and the claim is proved.

\section{EXAMPLES}

In this section we continue to compare the Weierstrass elliptic function with corresponding members of the family $f_{\gamma}$ with an explicit example. For lattices with extra symmetry, the comparison is quite good. We consider the Weierstrass $\wp$ function $\wp_{\Lambda}$ using $\left(g_{2}, g_{3}\right)=(0,6)$. From [11] (see also 9]) we know the following about $\wp_{\Lambda}$ :

Example 4.1. The function $\wp_{\Lambda}$ using $\left(g_{2}, g_{3}\right)=(0,6)$ satisfies

(1) $\wp_{\Lambda}(z)=\frac{1}{z^{2}}+\frac{3}{14} z^{4}+O\left(z^{10}\right)$ (all coefficients of $z^{m}, m \geq 10$ are zero unless $m+2$ is divisible by 6 ). 

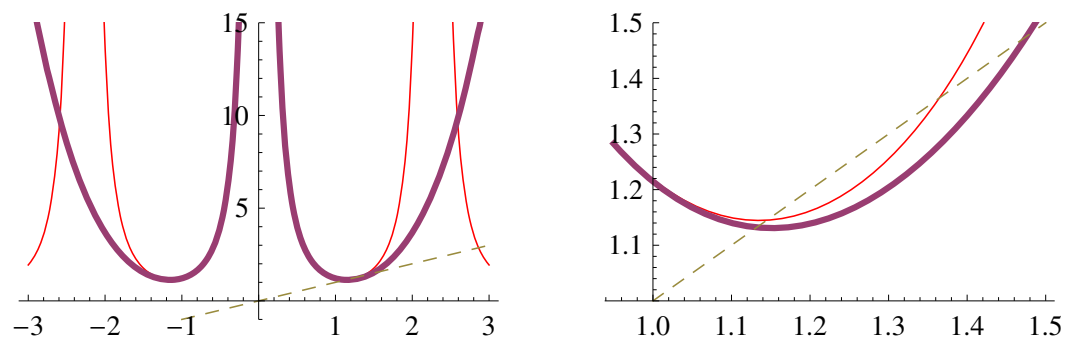

FIGURE 1. The graphs of $\wp_{\Lambda}$ (thin), $f_{4,2,3 / 14}$ (thick), and $x=y$ (dotted line) near 0

(2) The period lattice $\Lambda(0,6)$ is real triangular (a fundamental period is a quadrilateral made from 2 equilateral triangles, with symmetry about the real axis); therefore $F\left(\wp_{\Lambda}\right)=e^{2 \pi i / 3} F\left(\wp_{\Lambda}\right)$.

(3) The three critical values are the cube roots of $g_{3} / 4=3 / 2$; they are $e_{0} \approx$ 1.1447, $e_{1}=e^{2 \pi / 3} e_{0}$, and $e_{2}=e^{4 \pi / 3} e_{0}$.

(4) If $\Lambda$ has side length $\lambda>0$, then there are six critical points symmetric about 0 at all half lattice points, which are located approximately at: $c_{0} \approx$ 1.13498, $c_{1}=e^{2 \pi / 3} c_{0}, c_{2}=e^{4 \pi / 3} c_{0}$, and $c_{4}=-c_{0}, c_{5}=-c_{1}, c_{6}=-c_{2}$.

(5) $\wp_{\Lambda}$ has attracting fixed points at $p_{0} \approx 1.14512, p_{1}=e^{2 \pi / 3} p_{0}$, and $p_{2}=$ $e^{4 \pi / 3} p_{0}$ and is structurally stable if we vary $\left(g_{2}, g_{3}\right)$. We have real derivatives $\wp_{\Lambda}^{\prime}\left(p_{j}\right) \approx .08$ for each $j$.

We make parallel statements for the map $\mathcal{P}_{4,2,3 / 14}$, which is conformally conjugate to $f_{4,2,3 / 14}$, by Lemma 1.1

Example 4.2. For the map $\mathcal{P}_{4,2,3 / 14}$ :

(1) There are three distinct critical values at $e_{0}=\frac{3}{2}\left(\frac{3}{7}\right)^{1 / 3} \approx 1.1309, e_{1}=$ $e^{2 \pi / 3} e_{0}$, and $e_{2}=e^{4 \pi / 3} e_{0}$.

(2) There are 6 nonzero critical points in $\mathbb{C}$ located approximately at: $c_{0} \approx$ 1.1517, $c_{1}=e^{2 \pi / 3} c_{0}$, and $c_{2}=e^{4 \pi / 3} c_{0}$; the remaining 3 are the negatives of these.

(3) $\mathcal{P}_{4,2,3 / 14}$ has attracting fixed points at $p_{0} \approx 1.1322, p_{1}=e^{2 \pi / 3} p_{0}$, and $p_{2}=$ $e^{4 \pi / 3} p_{0}$ and is structurally stable if we vary $\frac{3}{14}$. We have real derivatives $\mathcal{P}^{\prime}\left(p_{j}\right) \approx-.134$ for each $j$.

Obviously for the conformally conjugate map $f_{4,2,3 / 14}$ we have the same properties at slightly different locations.

We show the graphs of these two maps near 0 in Figure1. Illustrations for results in this paper about Julia sets appear on the author's website.

\section{REFERENCES}

[1] Alan F. Beardon, Iteration of rational functions, Graduate Texts in Mathematics, vol. 132, Springer-Verlag, New York, 1991. Complex analytic dynamical systems. MR.1128089 (92j:30026)

[2] Walter Bergweiler, Iteration of meromorphic functions, Bull. Amer. Math. Soc. (N.S.) 29 (1993), no. 2, 151-188, DOI 10.1090/S0273-0979-1993-00432-4. MR.1216719 (94c:30033) 
[3] P. Blanchard, F. Cilingir, D. Cuzzocreo, D. Look, and E. Russell, Checkerboard Julia Sets for Rational Maps (preprint, 2011).

[4] Joshua J. Clemons, Dynamical properties of Weierstrass elliptic functions on square lattices, ProQuest LLC, Ann Arbor, MI, 2010. Thesis (Ph.D.)-The University of North Carolina at Chapel Hill. MR2941395

[5] Joshua J. Clemons, Connectivity of Julia sets for Weierstrass elliptic functions on square lattices, Proc. Amer. Math. Soc. 140 (2012), no. 6, 1963-1972, DOI 10.1090/S0002-99392011-11079-7. MR2888184

[6] Robert L. Devaney, Structure of the McMullen domain in the parameter planes for rational maps, Fund. Math. 185 (2005), no. 3, 267-285, DOI 10.4064/fm185-3-5. MR2161407 (2006c:37046)

[7] Robert L. Devaney, Daniel M. Look, and David Uminsky, The escape trichotomy for singularly perturbed rational maps, Indiana Univ. Math. J. 54 (2005), no. 6, 1621-1634, DOI 10.1512/iumj.2005.54.2615. MR2189680(2006i:37105)

[8] R. Devaney, E. Russell, Connectivity of Julia Sets for Singularly Perturbed Rational Maps (preprint, 2012).

[9] Patrick Du Val, Elliptic functions and elliptic curves, Cambridge University Press, London, 1973. London Mathematical Society Lecture Note Series, No. 9. MR0379512 (52:417)

[10] Jane Hawkins, A family of elliptic functions with Julia set the whole sphere, J. Difference Equ. Appl. 16 (2010), no. 5-6, 597-612, DOI 10.1080/10236190903257859. MR2642468 (2011f:37080)

[11] Jane Hawkins and Lorelei Koss, Ergodic properties and Julia sets of Weierstrass elliptic functions, Monatsh. Math. 137 (2002), no. 4, 273-300, DOI 10.1007/s00605-002-0504-1. MR.1947915 (2003j:37066)

[12] Jane Hawkins and Lorelei Koss, Parametrized dynamics of the Weierstrass elliptic function, Conform. Geom. Dyn. 8 (2004), 1-35 (electronic), DOI 10.1090/S1088-4173-04-00103-1. MR2060376 (2005b:37089)

[13] Jane Hawkins and Lorelei Koss, Connectivity properties of Julia sets of Weierstrass elliptic functions, Topology Appl. 152 (2005), no. 1-2, 107-137, DOI 10.1016/j.topol.2004.08.018. MR:2160809 (2007b:37098)

[14] Jane Hawkins and Mark McClure, Parameterized dynamics for the Weierstrass elliptic function over square period lattices, Internat. J. Bifur. Chaos Appl. Sci. Engrg. 21 (2011), no. 1, 125-135, DOI 10.1142/S0218127411028301. MR2786816(2012d:37091)

[15] John Milnor, Dynamics in one complex variable, Friedr. Vieweg \& Sohn, Braunschweig, 1999. Introductory lectures. MR 1721240 (2002i:37057)

[16] Yingqing Xiao and Weiyuan Qiu, The rational maps $F_{\lambda}(z)=z^{m}+\lambda / z^{d}$ have no Herman rings, Proc. Indian Acad. Sci. Math. Sci. 120 (2010), no. 4, 403-407, DOI 10.1007/s12044010-0044-x. MR2761768(2012b:37124)

Department of Mathematics, University of North Carolina at Chapel Hill, CB \#3250, Chapel Hill, North Carolina 27599-3250

E-mail address: jmh@math.unc.edu 